\title{
An investigation and statistical analysis into the incidents and failures associated with dynamic positioning systems
}

\author{
O. Olubitan, S. Loughney \& J. Wang \\ Liverpool John Moores University, Liverpool, UK \\ R. Bell \\ Engineering Safety Consultants Ltd., UK
}

\begin{abstract}
This investigation highlights the safety of DP (Dynamic Positioning) systems by briefly discussing the existing risk assessment methods and risk control measures provided by the International Maritime Organization (IMO). Following a study regarding DP systems and the loss of position incidents reported to the International Marine Contractors Association (IMCA) and contained in the World Offshore Accident Databank (WOAD), the relevant hazards are identified and collated. The primary causes of loss of position incidents were found to be: the positional reference system failures and thruster failures, with both contributing to $20.6 \%$ each of the total incidents from 2000 to 2016. Similarly the time period of the analysis is the 17 years between 2000 and 2016. Given the two stated primary causes, the positional reference system failures and thruster failures, the thruster failures are analysed further. This is due to thruster failures occurring at an increased rate within DP incidents from 2011 to 2016. Hence, this trend, between 2011 and 2016, is analysed further. Three undesired events for loss of position due to thruster failures were identified, these are as follows: "drive off", "drift off" and "time loss". Further investigation of incidents in more recent years, in this case, 2012 to 2016, identified that the DP control system accounted for $33.7 \%$ of the initiating incidents that led to thruster failures. Furthermore, the undesired event "time loss" accounted for $71.2 \%$ of total incidents caused by thruster failures.
\end{abstract}

\section{INTRODUCTION}

Many marine vessels are now equipped with Dynamic Positioning (DP) systems. These vessels have different functions and come in different sizes. Some vessels are used for Simultaneous Operations (SIMOPS) and Combined Operations (COMOPS), bringing them in close proximity with other vessels and/or offshore installations. Some are used to conduct diving operations, with a huge number of vessels also used for drilling operations. The functions of these DP vessels are very numerous, challenging and distinct. To cater for these functions, their level of safety was required to be increased and classified. Through time, with support from different organizational bodies and from lessons learnt from past DP failures, the safety of DP systems and vessels has been increased. These DP systems now utilize a redundancy scheme to ensure that a single failure would not lead to an accident. In this research the term safety is derived from the definition of functional safety outlined by Bell (2010). This states that "functional safety is a part of the overall safety that depends on a system or equipment operating correctly in response to its inputs. In essence, this means the achievement of safety through application of control systems. This requires identifying what has to be done and how well it should be done" (Bell, 2010).

DP has evolved over the years, from use as a tool for mobile offshore drilling units, for maintaining position over offshore wells, to being employed for a wide range of position keeping operations. We see DP systems being fitted on an increasingly large number of new and diverse vessels, from offshore units to shuttle tankers to passenger vessels (IMO 1994).

The increase in the number of diverse applications results in an increase to the estimated risks involved with dynamic positioning vessels, further requiring an increased level of safety. To this end there has been a growth in the development of the dynamic positioning systems. There are now several classes from DP0 to DP3 (DNV 2012), each class with a different level of safety.

The rationale behind this topic is to identify, investigate and analyse the loss of position incidents due to DP system failure. These incidents are analysed 
according to the seasons in which they occurred, types of vessels, main causes and initiating causes.

\section{BACKGROUND}

\subsection{Dynamic positioning}

DP can be defined as "a means of holding a vessel in a relatively fixed position with respect to the ocean floor, without using anchors, accomplished by two or more propulsive devices, controlled by inputs from sonic instruments on the sea bottom and on the vessel, by gyrocompass, by satellite navigation or by other means" (Holvik 1998).

The launch of the Global Positioning System Satellite network brought new ideas and new technology to be integrated into the DP vessels for more efficient performance. In 1981, the Nautical Institute began working on certification process for DP operators. This was done to reduce accidents and failures caused by human error. In 1983, the Department of Energy and the Norwegian Petroleum Directorate produced guidelines for diving from DP vessels. Howard Shatto in 1983 further improved on the DP systems, allowing greater water depths of 7,500 $\mathrm{m}$ and even rougher seas to be achievable. From his knowledge of satellite positioning and through his participation in the first use of Failure Mode and Effects Analysis (FMEA) for DP systems in 1983, the Mean Time Between Failures (MTBF) for DP systems was improved six-fold (DPC-MTS 1996).

By 1985, the number of DP capable vessels had increased to over 150. At this time, the vessel types equipped with DP systems had also increased. The following are some of the types of DP vessels that were available by 1985 based on their functions: Drillships, Mobile Offshore Drilling Units (MODU), Diving Semi-Submersibles, Diving and Emergency Response Vessels, Remote Operated Vessels, Diving Support Vessels, Shuttle Tankers and Accommodation Vessels (Flotels).

The following years saw an increase in the use of DP systems various functions and vessel types. In 1990, the first DP Floating Production Storage and Offloading (FPSO) vessel, Seillean, was launched by British Petroleum. The Dynamic Positioning Vessel Owners Association (DPVOA) was formed in the same year. In 1994, IMO provided guidelines for DP systems, MSC/Circ.645, the same year, the American Bureau of Shipping (ABS), introduced their first DP rules. The following year, 1995, the International Marine Contractors Association (IMCA) was formed through the merger of the Association of Offshore Diving Contractors and the DPVOA. In a bid to encourage exchange of information, foster improvement of DP reliability, develop guidelines, train and educate, and address any other issues pertinent to DP that encourage an incident free operation of DP systems. The DP committee was founded in 1996 as a Professional Committee of the Marine Technology Society (Sean 2009).

DP systems have been improved considerably since the installation of the first DP drill ship, Eureka. Vessels used for a variety functions are now equipped with DP systems. Some functions have been stated previously.

\subsection{Classification of the DP systems}

The different classes in the IMO and the ABS regulations are examined. The only difference between the two is that there is not a Class 0 for IMO regulations. ABS uses DPO to refer to vessels without DP systems. IMO does not put this as a class of DP vessels. IMO classes start from Class 1.

\section{Class 0: DPS-0}

"For vessels, which are fitted with centralized manual position control and automatic heading control system to maintain the position and heading under the specified maximum environmental conditions" (ABS, 2013). Class 0 does not exist on the IMO classification.

2. Class 1: DPS-1

"For vessels, which are fitted with a dynamic positioning system, which is capable of automatically maintaining the position and heading of the vessel under specified maximum environmental conditions having a manual position control system" (ABS, 2013).

3. Class 2: DPS-2

"For vessels, which are fitted with a dynamic positioning system, which is capable of automatically maintaining the position and heading of the vessel within a specified operating envelope under specified maximum environmental conditions during and following any single fault, excluding a loss of compartment or compartments" (ABS, 2013).

4. Class 3: DPS-3

"For vessels, which are fitted with a dynamic positioning system, which is capable of automatically maintaining the position and heading of the vessel within a specified operating envelope under specified maximum environmental conditions during and following any single fault, including complete loss of a compartment due to fire or flood" (ABS, 2013).

DPS-1, DPS-2 and DPS-3 classification notations stated by ABS (2013) are structured to conform to IMO (2017). Therefore, the classifications DPS-1, DPS-2 and DPS-3 relate to IMO's equipment classifications 1, 2 and 3 (ABS, 2013). 


\section{STATISTIC ANALYSIS}

For the purpose of hazard identification, incident data has been gathered from two sources: the World Offshore Accident Database (WOAD) and the IMCA. The data from IMCA has been utilised for the majority of the analysis as it is consistent and covers a substantial time period.

\subsection{Terms used by IMCA}

The incidents and events listed in the various reports have been categorized by IMCA into three areas, where PL stands for Position Loss. These categories are:

1. DP Incident (PL 1): This is the loss of automatic $D P$ control, loss of position or any other incident which has resulted in or should have resulted in a RED Alert status (IMCA 2017). In other words, these are incidents of a serious nature.

2. DP Undesired Event (PL 2): Loss of position, loss of stability, or another event which is unexpected or uncontrolled and has resulted in or should have resulted in a Yellow Alert status (IMCA 2017). As such, they are incidents of a less serious nature.

3. DP Downtime: Position keeping problem or loss of redundancy which would not warrant either a 'Red' or 'Yellow' alert, but where loss of confidence in the DP has resulted in a standdown from operational status for investigation, rectification, trials, etc. (IMCA 2017). From an operational point of view, a loss of time or downtime can be seen as an undesired event which should be avoided at all cost, so as to save money.

For the purpose of this research, further analysis focuses on the prior two incidents; DP Incidents (PL 1) and Undesired Incidents (PL 2).

There are two common types of position loss, these are as follows:

1. Drive Off: This is characterised by the thrusters going to high unwanted thrust usually because the DP control system believes the position is wrong (Jenman 1998).

2. Drift Off: This is caused by the lack of sufficient power or thrust. For example, a total blackout on a ship on high seas, combined with strong currents. This would lead to a drift off (Jenman 1998).

There was a debate as to how one would differentiate a "drift off" and a "drive off" of vessels that were quickly recovered and returned to their original position as opposed to vessels which travelled far from their original position and could not be easily recovered (Jenman 1998). These arguments led to the addition of the third type of position loss, Large Excursion.

3. Large Excursion: This is an excursion that takes the DP vessel beyond its normal excursion characterised by its footprint. The footprint is the outline of the vessels movement in a particular sea state (Jenman 1998).

\subsection{IMCA reporting}

Vessels from all over the world, report DP related incidents to IMCA, who ensure that the vessel reporting is kept anonymous, thereby ensuring safety and keeping company integrity. Also, there are a range of export regulations and restrictions which affect business and trade with many countries of the world. These include restrictions on dual-goods and technology as well as the various sanctions regimes (UN, US and EU) targeting individual states including, regulation (EU) No. $833 / 2014$ which is directed at Russia.

The data used in this research is available on the IMCA website, to IMCA members only. However, there are exceptions so that the information can be released to other interested parties who are not IMCA members. IMCA requires both its members and non-members to confirm that they will not use any IMCA document in breach of the Restrictions.

The data provided spanned over 17 years (2000-2016). From the reporting styles over the years, some improvements in reporting are visible, thereby creating differences in the data provided.

From Table 1, a total of 1,163 incidents were analysed and documented by IMCA between the 17 -year periods. From Table 1, it is possible that the incident reporting by vessel owners is not consistent. It declined from 2000 to 2004 then picked back up, to peak in 2008 and fall again in 2010 . Then there was a steady increase throughout the remaining years.

More emphases should be placed on incident reporting, including potential near misses.

Table 1. Incident data analysed from 2000 to 2016.

\begin{tabular}{lrrr}
\hline \multicolumn{2}{l}{ Incidents reported } & & \\
\hline 2000 & 110 & 2008 & 102 \\
2001 & 98 & 2009 & 75 \\
2002 & 64 & 2010 & 56 \\
2003 & 51 & 2011 & 54 \\
2004 & 34 & 2012 & 64 \\
2005 & 36 & 2013 & 64 \\
2006 & 59 & 2014 & 71 \\
2007 & 67 & 2015 & 80 \\
2008 & 102 & 2016 & 78
\end{tabular}


From the 1,163 incidents analysed, 633 of those incidents indicated the month in which they occurred. This was from the year 2007 to 2015. Based on these IMCA reports, Table 2 was developed.

It can be seen from Table 2 that in the spring and summer of the 9 years, recorded the highest amount of incidents reported. Taking a closer look at the individual years and months, it is evident that even though spring has the highest number of incidents reported over the 9 years, there were more years when summer had more incidents reported than spring. From this, one can state that the majority of the incidents reported occur during summer and spring time.

Looking at the seasons of the year and looking at the months where natural disasters are prominent, this falls within the same seasons as the one stated above. According to AccuWeather Inc., an American media company that provides commercial weather forecasting services worldwide, hurricanes occurs at different times of the year for different regions in the world. For the Atlantic Ocean, hurricane season runs from June to November. In the Eastern Pacific Ocean, it occurs in the months of May through to November. The hurricane season for the Western Pacific Ocean runs from July to November, while in the South Pacific Ocean, it runs from October through to May, reaching a peak in late February or early March. The Indian Pacific Ocean's hurricane season runs from April to December in the Northern Indian Ocean and October to May in the Southern region (Mummey 2010).

Research conducted by the University of Manchester, showed that tornado season in the UK occurs within the months of May to October (Mulder \& Schultz 2015). Mother Nature Network on tornado season in America stated that it occurs between March and Early June in the Southern regions. They also state that in the Gulf Coast, tornadoes occur during the spring, while peaking in June and July in the Northern regions. Some states are also mentioned to experience a later tornado season from October to December (Sarah 2011).

From the analyses above, it is evident that most of the adverse weather conditions occur during the spring to autumn seasons. It is safe to attribute this to the reason why there is a higher frequency of incidents reported within the spring to autumn seasons.

Furthermore, the IMO does not state at which time of the day these incidents have occurred. Similarly, it should be noted that these incidents are provided from all around the globe, not one specific region.

For legal, trade and restriction reasons, the number of incidents occurring in different areas of the world has been withheld.

\subsection{Incidents according to vessel type}

Several DP vessels with different functions report incidents to IMCA. Inconsistency in reporting and change of reporting style can be seen in this area. The years stated in this report are the years that were clearly documented by IMCA. The following types of vessels that reported incidents over the aforementioned years are as follows:

1. Remote Operated Vehicles (ROVs)

2. DP Diving Support Vessels (DSV)

3. Drilling Vessels

4. Pipe/Cable Lay Vessels

5. Offshore Loading Vessels

6. Standby Vessels

7. Well Operations Vessels

8. Seismic Vessels

9. Multi Service Vessels (MSV)

10. Shuttle Tanker

Table 2. Incident data analysed from 2000-2016.

\begin{tabular}{lrrrrrrrrrrrr}
\hline & 2007 & 2008 & 2009 & 2010 & 2011 & 2012 & 2013 & 2014 & 2015 & Total & Season \\
\hline Dec & 10 & 10 & 7 & 3 & 11 & 1 & 2 & 3 & 6 & 53 & 136 & Winter \\
Jan & 5 & 6 & 4 & 1 & 5 & 4 & 6 & 5 & 4 & 40 & \\
Feb & 6 & 2 & 5 & 10 & 2 & 4 & 2 & 5 & 7 & 43 & \\
Mar & 5 & 7 & 7 & 4 & 5 & 10 & 8 & 7 & 10 & 63 & 179 & Spring \\
Apr & 6 & 7 & 12 & 4 & 2 & 6 & 3 & 8 & 10 & 58 & & \\
May & 9 & 14 & 3 & 3 & 5 & 2 & 8 & 8 & 6 & 58 & \\
Jun & 4 & 11 & 8 & 6 & 5 & 8 & 11 & 5 & 7 & 65 & 172 & Summer \\
Jul & 5 & 7 & 7 & 5 & 4 & 7 & 6 & 5 & 7 & 53 & & \\
Aug & 6 & 11 & 9 & 5 & 3 & 3 & 5 & 8 & 4 & 54 & \\
Sep & 6 & 9 & 3 & 6 & 4 & 4 & 6 & 5 & 5 & 48 & 145 & Autumn \\
Oct & 4 & 7 & 2 & 4 & 2 & 10 & 3 & 7 & 5 & 44 & \\
Nov & 1 & 11 & 7 & 5 & 6 & 5 & 4 & 5 & 9 & 53 & \\
Total & 67 & 102 & 74 & 56 & 54 & 64 & 64 & 71 & 80 & 632 & \\
\hline
\end{tabular}


11. Flotels

12. Construction Vessels

13. FPSO

14. Rock Dumping Vessels

15. Crane Vessels

16. Supply Vessels

Since there are different types of vessels of different functions and not all these vessels reported incidents every year, Table 3 shows a list of the vessels that reported incidents consistently through the stated time period.

Table 3 shows that DSVs have reported the most incidents in the 10 years stated above. From 2008 to 2014 , the reporting style changed and the various vessels that had experienced incidents were not reported.

Table 3. Types of vessels reported 2000-2016.

\begin{tabular}{lccc}
\hline & $\begin{array}{c}\text { DP } \\
\text { DSVs }\end{array}$ & Drilling & $\begin{array}{l}\text { Pipe/Cable } \\
\text { Lay }\end{array}$ \\
\hline 2000 & 21 & 22 & 4 \\
2001 & 35 & 5 & 15 \\
2002 & 11 & 5 & 12 \\
2003 & 15 & 10 & 4 \\
2004 & 4 & 13 & 2 \\
2005 & 9 & 15 & 2 \\
2006 & 13 & 9 & 10 \\
2007 & 16 & 15 & 11 \\
2015 & 10 & 20 & 9 \\
2016 & 18 & 2 & 19 \\
Total & 152 & 116 & 88 \\
\hline
\end{tabular}

\subsection{Incident main causes}

In the IMCA reports, they go further by indicating the primary cause of each incident, making the statistical analysis very easy for the user. Table 4 shows the incident causes over the past few years

As shown in Table 4, the amount of incidents caused by the various DP elements are visible. On further study of Table 4, the elements that cause the most incidents per year can be identified. These elements have been highlighted in bold in Table 4. Judging from this, it is evident that the major main causes of the DP incidents that have been reported are caused by either Reference element or the Thruster Element. It should be noted that the reference element mentioned in Table 4 includes the sensors while the thruster element mentioned includes the propulsion system.

It has been found that in the years prior to 2010, reference system failures were the main cause of incidents. However, from 2011 to 2016, it can been seen that thruster failures have consistently been identified as the main cause of DP incidents. In 2000 , there was a drastic drop in incidents related to reference systems. The number of reference related incidents then peaks again in 2008 falls drastically again up to 2016 and the reasons for this has not been identified here. It may be assumed that multiple DP regulations were adopted and enforced across the 17-year period which led to the decrease in reference related incidents.

Looking at the whole 17-year period, Table 4 sums up the total incidents caused by the different

Table 4. Incident main causes from 2000-2016.

\begin{tabular}{lccccccccc}
\hline Main causes & Computer & Environment & Power gen. & Operator & Reference & Thruster & Electrical & Other & Total \\
\hline 2000 & 18 & 5 & 12 & 18 & $\mathbf{3 4}$ & 17 & 3 & 3 & 110 \\
2001 & $\mathbf{2 3}$ & 18 & 8 & 8 & 14 & 14 & 10 & 3 & 98 \\
2002 & 11 & 3 & 13 & 8 & $\mathbf{1 4}$ & 12 & 3 & 0 & 64 \\
2003 & 4 & 7 & 8 & $\mathbf{1 4}$ & 6 & 11 & 0 & 1 & 51 \\
2004 & 1 & 2 & 8 & 6 & $\mathbf{1 2}$ & 4 & 1 & 0 & 34 \\
2005 & $\mathbf{8}$ & 3 & 4 & 5 & 5 & 7 & 4 & 0 & 36 \\
2006 & 7 & 4 & 12 & $\mathbf{1 3}$ & 11 & 4 & 6 & 2 & 59 \\
2007 & $\mathbf{1 8}$ & 4 & 11 & 7 & 13 & 8 & 5 & 1 & 67 \\
2008 & 22 & 3 & 9 & 5 & $\mathbf{2 7}$ & 21 & 10 & 5 & 102 \\
2009 & 8 & 2 & 13 & 10 & $\mathbf{1 8}$ & 12 & 10 & 2 & 75 \\
2010 & 6 & 4 & 5 & 3 & $\mathbf{2 1}$ & 2 & 12 & 3 & 56 \\
2011 & $\mathbf{1 4}$ & 5 & 7 & 3 & 9 & 13 & 3 & 0 & 54 \\
2012 & 8 & 2 & 6 & 11 & 11 & $\mathbf{2 0}$ & 4 & 2 & 64 \\
2013 & 6 & 3 & 13 & 7 & 15 & $\mathbf{2 0}$ & 0 & 0 & 64 \\
2014 & 13 & 2 & 9 & 7 & 12 & $\mathbf{2 6}$ & 0 & 2 & 71 \\
2015 & 13 & 11 & 10 & 10 & 11 & $\mathbf{2 4}$ & 1 & 0 & 80 \\
2016 & 15 & 0 & 15 & 16 & 7 & $\mathbf{2 4}$ & 0 & 1 & 78 \\
Incidents & 195 & 78 & 163 & 151 & $\mathbf{2 4 0}$ & $\mathbf{2 3 9}$ & 72 & 25 & 1163 \\
Percentage & $16.8 \%$ & $6.7 \%$ & $14.0 \%$ & $13.0 \%$ & $\mathbf{2 0 . 6} \%$ & $\mathbf{2 0 . 6} \%$ & $6.2 \%$ & $2.1 \%$ & $100 \%$ \\
\hline
\end{tabular}


systems and elements, showing their various percentages as they relate to each other.

\subsection{Thruster statistics analysis}

From the analysis of Table 4, it is evident that the thruster system in recent times (2012-2016) has been the major cause of incidents. Looking further into this, it is possible to define initiating causes of undesired events relating to thruster failures. For this, a more specific analysis of incidents caused by thruster failures, that occurred within the years of 2012-2016, was conducted. It can be seen that for the period in question, a total of 357 incidents occurred, of which, 114 incidents were thruster-caused $(31.9 \%)$, which is extremely high compared to other elements. Of the 114 incidents, 105 were categorized into the different undesired events; "drift off", "drive off" and "time loss" events. Table 5 shows the undesired incidents that occurred and their initiating events.

From Table 5, it is clear that the majority of thruster related incidents begin with a fault in the DP control system, followed by the electrical system and the mechanical system. It is worth noting here that some minor failures have been grouped together to make up the failures mentioned above. For control system, there are failures such as, feedback error, loss of control, wrong DP operator input, etc. These are the failures that are rectified when the DP control system is restarted. Software errors can also fall under DP control system errors. Under electrical errors, there are occurrences such as: loose wiring, fibre optic fault, low/high voltage supply, loose fuse, field circuit failure and DC motor failure. In this case, when the loose wire has been fixed or the electrical problem fixed, operation continues.

In the case of mechanical initiating failures, there are faults such as, low oil pressure, hydraulic pump failure, faulty valve, engine failure, oil pipe leakage, cooling motor failure, brake failure, etc. In any of these mechanical event sce-

Table 5. Undesired incidents identified from thruster failures and their initiating causes from 2012 to 2016 .

\begin{tabular}{lcccr}
\hline $\begin{array}{l}\text { Undesired } \\
\text { events }\end{array}$ & $\begin{array}{l}\text { Drive } \\
\text { off }\end{array}$ & $\begin{array}{l}\text { Drift } \\
\text { off }\end{array}$ & $\begin{array}{l}\text { Time } \\
\text { loss }\end{array}$ & Total \\
\hline Reference & 0 & 0 & 2 & 2 \\
Electrical & 5 & 3 & 21 & 29 \\
Mechanical & 2 & 2 & 21 & 25 \\
Generator & 0 & 0 & 1 & 1 \\
Control system & 7 & 6 & 22 & 35 \\
Human error & 3 & 2 & 7 & 12 \\
Total & 17 & 13 & 74 & 104 \\
\hline
\end{tabular}

narios, a redundant system will have to be used or the system will be taken off DP control and returned to port for fixing. For the category of human error, there are a number human failures, such as: wrong procedures, lack of maintenance, inexperience and late response. Some human errors fall under the DP control system, because there has to be an interface between the control system and the human. Here, depending on the fault, the vessel is regained as soon as possible to avoid a worse undesired event. For the reference system, there are errors such as; wind sensor errors, tachometer errors, DGPS errors, etc. These errors coupled with some hidden errors can cause the thruster to fail at an odd angle (pitch). Finally, there are failures relating to the power generators. These failures are explanatory on their own and can cause more than the thrusters to fail. They also include the failure of the Uninterrupted Power Supply system (UPS). When these fail, they can cause an immediate failure of the thruster, which can be fixed by a redundant power generation system.

\section{CONCLUSION}

From the statistical analysis presented in this study, it was possible to determine the number of incidents that occurred in the different seasons of the year, over a 9-year period. From the results obtained, it was observed that there was an increase in the incidents in the spring and summer seasons. From research it was discovered that this could be as a result of the harsh natural weather conditions that occur during those seasons of the year, such as, tornadoes, hurricanes, etc. The tides that occur during the spring, resulting in high tide were also mentioned as a factor for increased incidents during these seasons.

The vessel types with the most incidents were also analysed. It was found that of all the vessels recorded, the three types; DSV, Pipelay and Drilling Vessels had the most incidents recorded. The DSV had the most recorded incidents, with 152 consistently recorded (see Table 3 ). The reason for this was not fully identified, however it can be argued that pipelay vessels are not used as frequently the DSVs or Drilling vessels. Hence, this has resulted in large difference in the number of reported incidents relating to these vessel types.

Following this, the main causes were analysed and separated into several categories. It was found that the two categories with the highest number of incidents in the 17 year period (2000-2016) were the reference and thruster systems. On further investigation of the data, it was found that within 
the years 2000 to 2002 there was a drastic drop in reference caused incidents. Although not found, it is suspected that a regulation was brought into force during that time that either checked incident occurrence or made the operators not to be able to report incidents.

Failure of thruster systems were further analysed because in previous years (2012-2016), they were the major cause of DP incidents. The analysis led to the discovery of the initiating events of the thruster failures and their final consequences (Drift-Off, Drive-Off and Time Loss). This helped to identify the hazards and establish the basic events that would cause the release of such hazards and provided a base to conduct a risk assessment.

Although there were changes in the style of reporting, which led to the inconsistency of some information, the data sourced from IMCA was sufficient in identifying the hazards relating to DP operations.

Finally, from the hazard analysis, reference failures and thruster failures were identified as the major causes of DP incidents, with control failure being the most frequent initiating event leading to thruster failures.

\section{ACKNOWLEDGEMENTS}

This work is partially supported by an EU Marie Curie RISE project RESET (reference no. 730888). Similarly, funding has been supplied by the Royal Academy of Engineering under reference number VP1415/1/22.

\section{DISCLAIMER}

This paper is the opinion of the authors and does not necessarily represent the belief and policy of their employers.

\section{REFERENCES}

ABS (American Bureau of Shipping), 2013. Guide for Dynamic Positioning Systems, November 2013 (updated July 2014), ABS, Houston, Texas.

Bell, R., 2010. Assessment, certification and other assurance measures. Engineering Safety Consultants Limited, IEC61508.

DNV, 2012. Dynamic Positioning Systems. Rules for Classification of Ships. Available at: www.dnv.com.

DPC-MTS, 1996. Dynamic Positioning Timeline. Available at: http://dynamic-positioning.com/timeline/ [Accessed June 5, 2017].

Holvik, J., 1998. Basics of Dynamic Positioning. MTS Dynamic Positioning Conference. Available at: http:// dynamic-positioning.com/proceedings/dp1998/BHolvik.PDF.

IMCA, 2017. International Marine Contractors Association-Statistics Archives. Available at: https:// www.imca-int.com/briefingseries/statistics/ [Accessed July 4, 2017].

IMO, 2017. International maritime organizationGuidelines for Vessels and Units with Dynamic Positioning (DP) Systems. MSC.1/Circ.1580, (June).

Jenman, C., 1998. Quantification of the Frequency of an Unsuccessful Disconnection because of a DP Problem. MTS Dynamic Positioning Conference. Available at: http://dynamic-positioning.com/proceedings/ dp1998/RJenman.PDF.

Mulder, K.J. \& Schultz, D.M., 2015. Climatology, Storm Morphologies, and Environments of Tornadoes in the British Isles: 1980-2012. Monthly Weather Review, 143(6), pp. 2224-2240. Available at: http://journals.ametsoc.org/doi/10.1175/MWR-D-14-00299.1 [Accessed August 22, 2017].

Mummey, 2010. When and Where Do Hurricanes Occur? AccuWeather. Available at: https://www.accuweather. com/en/weather-blogs/hurricanefacts/when-and-wheredo-hurricanes-o/31028 [Accessed August 22, 2017].

Sarah, B., 2011. When is tornado season?|MNN - Mother Nature Network. Mother Nature Network. Available at: https://www.mnn.com/family/protection-safety/stories/ when-is-tornado-season [Accessed August 22, 2017].

Sean, 2009. A Brief History of Dynamic Positioning Captain. Available at: http://gcaptain.com/history/ [Accessed May 25, 2017]. 